Recent Insights into the Physics of the Sun and Heliosphere:

Highlights from SOHO and Other Space Missions

IAU Symposium, Vol. 203, 2001

P. Brekke, B. Fleck, and J. B. Gurman eds.

\title{
Origin of Universal Correlation between Temperature and Emision Measure for Solar/Stellar Flares
}

\author{
K. Shibata \\ Kwasan Observatory, Kyoto University \\ T. Yokoyama \\ National Astronomical Observatory of Japan
}

We study the reconnection and the chromospheric evaporation in flares using the numerical code including nonlinear anisotropic heat conduction effect (Yokoyama \& Shibata 1998; 2001). The two-dimensional, nonlinear, timedependent, resistive, compressible MHD equations are solved. The evolution from the rise phase to (the early part of) the decay phase of a solar flare is qualitatively reproduced in this simulation. Based on the results, we obtained a relationship between the flare temperature and the coronal magnetic field strength. If we assume that the input of energy to a loop balances with the conduction cooling rate, the temperature at the loop apex is $T_{\mathrm{A}} \approx\left(2 Q L^{2} / \kappa_{0}\right)^{2 / 7}$ where $Q$ is the volumetric heating rate, $L$ is the half-length of the loop, and $\kappa_{0}=10^{-6}$ CGS is the Spitzer's thermal conductivity constant. In our simulations, the heating mechanism is magnetic reconnection so that the heating rate is described as $Q=B^{2} /(4 \pi) \cdot V_{\text {in }} / L \cdot 1 / \sin \theta$, where $B$ is the coronal magnetic field strength, $V_{\text {in }}$ is the inflow velocity $\left(\approx 0.1 V_{\mathrm{A}}\right.$ from our result and also from Petschek's theory), and $\theta$ is the angle between the slow-mode MHD shock and the loop and is approximately given by $\sin \theta \approx V_{\text {in }} / V_{\mathrm{A}}$. By manipulating the equations, we find

$$
T_{\mathrm{A}} \approx\left(\frac{B^{3} L}{2 \pi \kappa_{0} \sqrt{4 \pi \rho}}\right)^{\frac{2}{7}} \propto B^{\frac{6}{7}} \propto \beta^{-\frac{3}{7}},
$$

where $\rho$ is the mass density of the corona. The simulation results show very good agreement with this scaling law.

We also develop a theory to explain the observed universal correlation between flare temperature $T$ and emisssion measure $E M=n^{2} V$ for solar and stellar flares (including solar microflares observed by Yohkoh as well as protostellar flares observed by ASCA), where $n$ is the electron density and $V$ is the volume (Figure 1; Shibata \& Yokoyama 1999). The theory is based on the above magnetic reconnection model with heat coduction and chromospheric evaporation, assuming that the gas pressure of a flare loop is comparable to the magnetic pressure. This theory predicts the relation

$$
E M \propto B^{-5} T^{17 / 2}
$$




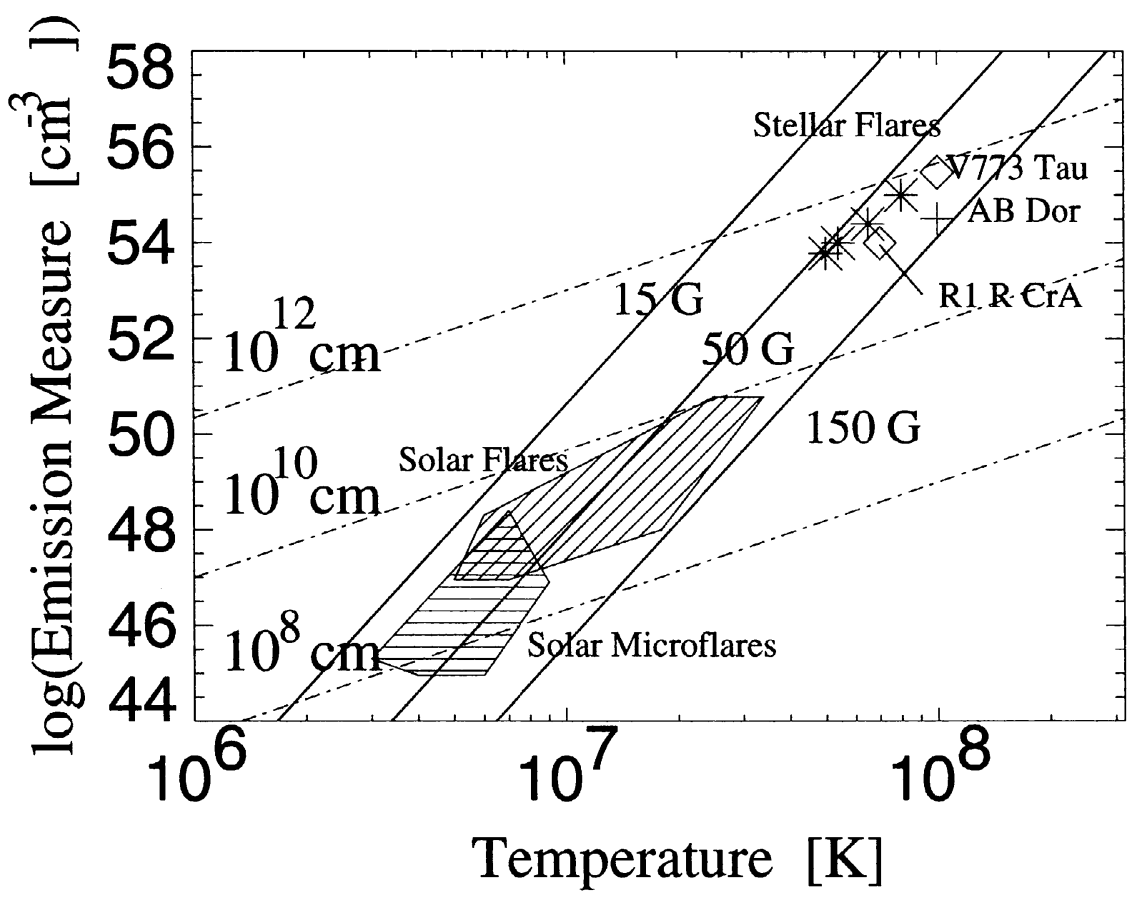

Figure 1. The log-log plot of emission measure vs. electron temperature of solar flares, solar microflares, four stellar flares (asterisks), a protostellar flare (diamond, class 1 protostar far IR source R1 in the R CrA cloud), a T-Tauri stellar flare (diamond, weaklined T-Tauri star V773 Tau), and a stellar flare on AB Dor (K0 IV ZAMS single star) The $E M-T$ relation curves $\left(E M \propto B^{-5} T^{17 / 2}\right)$ are superposed on the EM$\mathrm{T}$ diagram. The $L=$ constant curves (dashed lines; $E M \propto L^{5 / 3} T^{8 / 3}$ ) are also superposed on this diagram.

which explains well the observed correlation between $E M$ and $T$ in the range of $6 \times 10^{6} \mathrm{~K}<T<10^{8} \mathrm{~K}$ and $10^{44}<E M<10^{55} \mathrm{~cm}^{-3}$ from solar microflares to protostellar flares, if the magnetic field strength of a flare loop, $B$, is nearly constant for solar and stellar flares.

\section{References}

Shibata, K. \& Yokoyama, T. 1999 ApJ, 526, L49

Yokoyama, T. \& Shibata, K. 1998, ApJ, 494, L113

Yokoyama, T. \& Shibata, K. 2001, ApJ, 548, in press 